\title{
ALEXIS Lunar Observations
}

\section{B. C. EDWARDS, J. J. BLOCH, D. ROUSSEL-DUPRÉ, T. E. PFAFMAN, AND SEAN RYAN}

Mail Stop D436, Los Alamos National Laboratory, Los Alamos, NM, USA

The ALEXIS small satellite was designed as a large area monitor operating at extreme ultraviolet wavelengths $(130-190 \AA)$. At these energies, the moon is the brightest object in the night sky and was the first source identified in the ALEXIS data. Due to the design of $A L E X I S$ and the lunar orbit, the moon is observed for two weeks of every month. Since lunar emissions in the extreme ultraviolet are primarily reflected solar radiation these observations may be useful as a solar monitor in the extreme ultraviolet. The data show distinct temporal and spectral variations indicating similar changes in the solar spectrum. We will present a preliminary dataset of lunar observations and discussions covering the variations observed and how they relate to the solar spectrum.

\section{Introduction}

The Alexis experiment consists of three pairs of co-aligned extreme ultraviolet telescopes. Each telescope has a 33 degree field of view, narrow energy band response centered on 133,174 , or $188 \AA$ and one half degree spatial resolution. The $A L E X I S$ satellite is spin-stabilized with the telescopes pairs pointed 107 degrees (pair 3), 137 degrees (pair 2), and 167 degrees (pair 1) from the sun direction. In this orientation the telescopes observe half the sky during each spin period of roughly $50 \mathrm{~s}$. (For details on the Alexis experiment and satellite see Bloch et al. (1990) and Bloch this conference) This mission design provides a good platform for semi-continuous monitoring of extreme ultraviolet phenomenon.

In the case of the moon, Alexis semi-continually monitors its extreme ultraviolet emissions for two weeks around the full moon every lunar cycle. Since the lunar emission is primarily reflected solar radiation (Edwards et al. 1991; Gladstone et al. 1993) it provides a direct monitor of solar activity in the extreme ultraviolet. The primary solar lines that contribute to this part of the spectrum are from Fe X (primarily between 174 and $177 \AA$ ) and Fe XI (primarily between 180 and $190 \AA$ ) (Heroux 1974) which originate in the solar corona and photosphere. In addition to the reflected solar spectrum some of the lunar emission may be due to L- and M-shell fluorescence from the lunar surface (Edwards 1991).

\section{Discussion}

In determining the count rates associated with the moon we first converted the observed events into lunicentric coordinates and flagged events within 1.5 degree of the moon's position. We calculated the count rates by integrating the counts inside a 1 degree radius circle around the moon's position and using the remainder of the flagged events to determine the background rate (Figure 1). These rates were then corrected for the Alexis vignetting function. Complications in this calculation arose due to errors in the photon flagging routine and the aspect determination. Because these errors defocused the lunar image we chose an integration box much larger than the half degree resolution of Alexis would warrant. This insured all lunar photons were collected but it also reduced the signal to noise of our results. Future processing will correct these problems. For this processing we have also ignored variations in the intensity due to different phases of the 


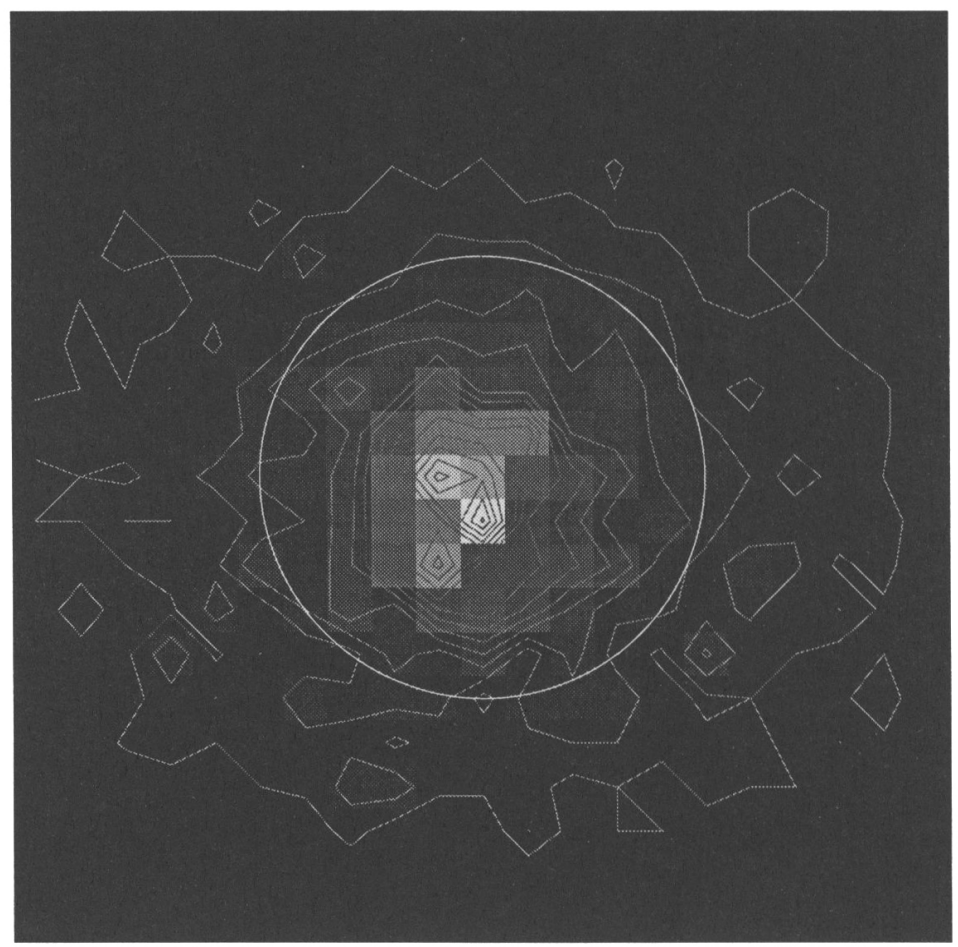

FIgURE 1. Image of the moon in lunicentric coordinates. The grayscale and contours show the relative counts observed. The circle shown is the $1^{\circ}$ radius area used to calculate the lunar count rate. Counts outside this circle were used to calculate the background rate.

moon. This will affect each pair of telescopes differently. In telescope pair three this will cause errors of less than 14 percent in the absolute rates but will not affect our rate ratio discussions.

The observed count rates varied from 0 to several counts per second depending on the telescope and time. Figures $2 a, 2 b$, and $2 c$ show averaged rates for over one year of Alexis observations. Each of the plotted data points represents the roughly 20 minutes of data obtained for each orbit of Alexis (see Figure 3a and $3 \mathrm{~b}$ ). The data have high statistics in some cases and show count rate variations of greater than two on the scale of hrs and greater than five on the scale of days. These rate variations can be compared to solar $10.7 \mathrm{~cm}$ indices (Figure 4) but no strong correlation is seen.

The ratio of the 188 and $174 \AA$ bands on telescope pair 3 can also be examined (Figure 5). The ratio is seen to vary primarily between 0.5 and 2 but statistically significant values are seen at all values. The ratio is not well correlated with the solar $10.7 \mathrm{~cm}$ indices (Figure 5). Since the $188 \AA$ band is primarily Fe XI and the $174 \AA$ band is primarily $\mathrm{Fe} X$, the ratio of these bands could be a very sensitive monitor of the relative Fe ion states that exist in the solar corona. The ion state and thus our rate ratio is a very sensitive solar corona temperature monitor in the range between 1 million and 1.5 million degrees. 

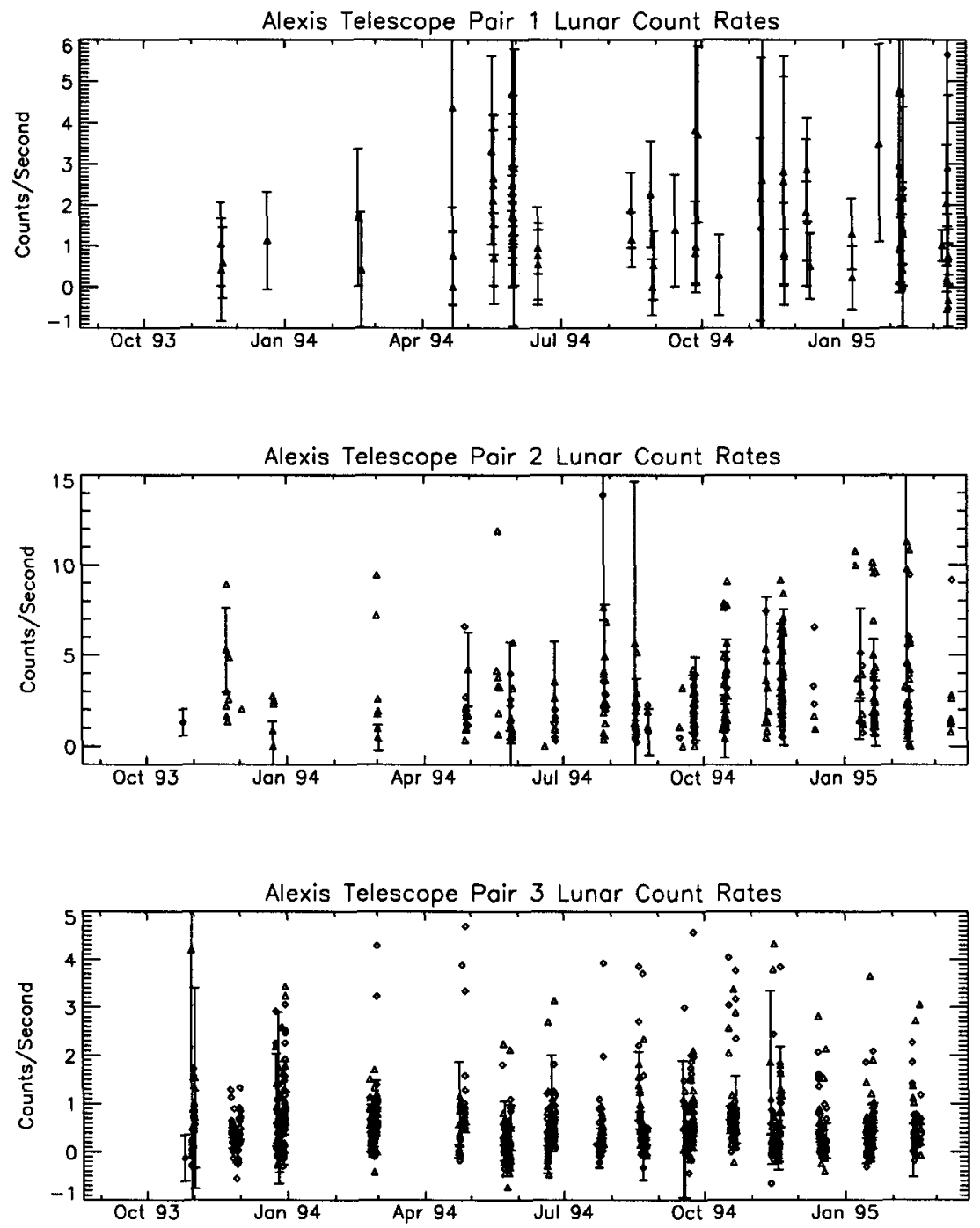

FIGURE 2. Lunar count rates observed by Alexis. a) Telescope pair 1, b) Telescope pair 2, c) Telescope pair 3. In all plots the diamonds are for the $A$ telescopes (1A:133 $\AA, 2 A: 133 \AA$, $3 A: 174 \AA$ ) and the triangles are for the B telescopes (1B: $174 \AA, 2 B: 188 \AA, 3 B: 188 \AA$ ). Several representative error bars are shown. 

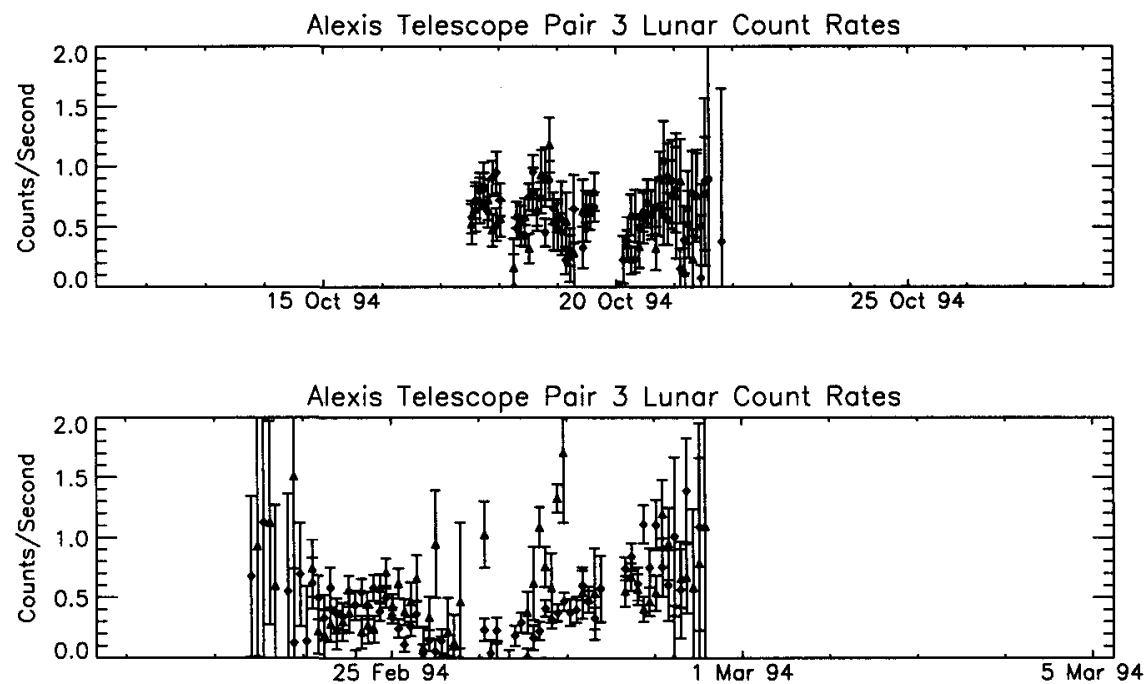

FIGURE 3. Lunar count rates observed by Alexis for several representative periods. The diamonds represent the $3 \mathrm{~A}$ telescope and the triangles represent the $3 \mathrm{~B}$ telescope. The count rates while in the center of the telescopes are observed to have good statistics. The rates determined for observations on the edge of the field of view rise due to error in the background determination.

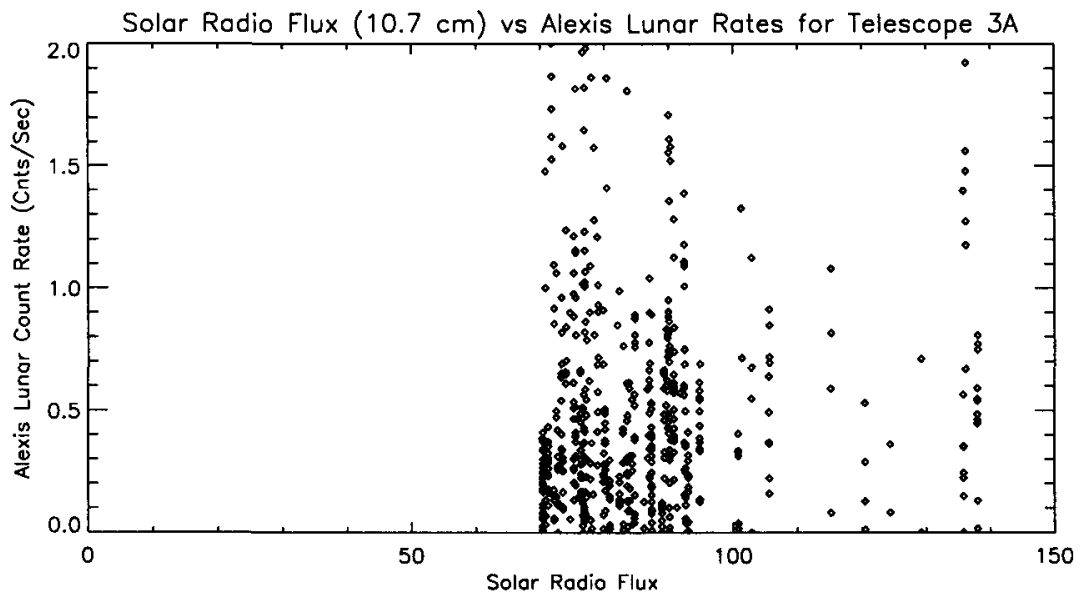

FIgURE 4. Solar $10.7 \mathrm{~cm}$ emission vs. Alexis lunar count rates (3A). Error bars are the same as plotted in Figures 1 and 2. 


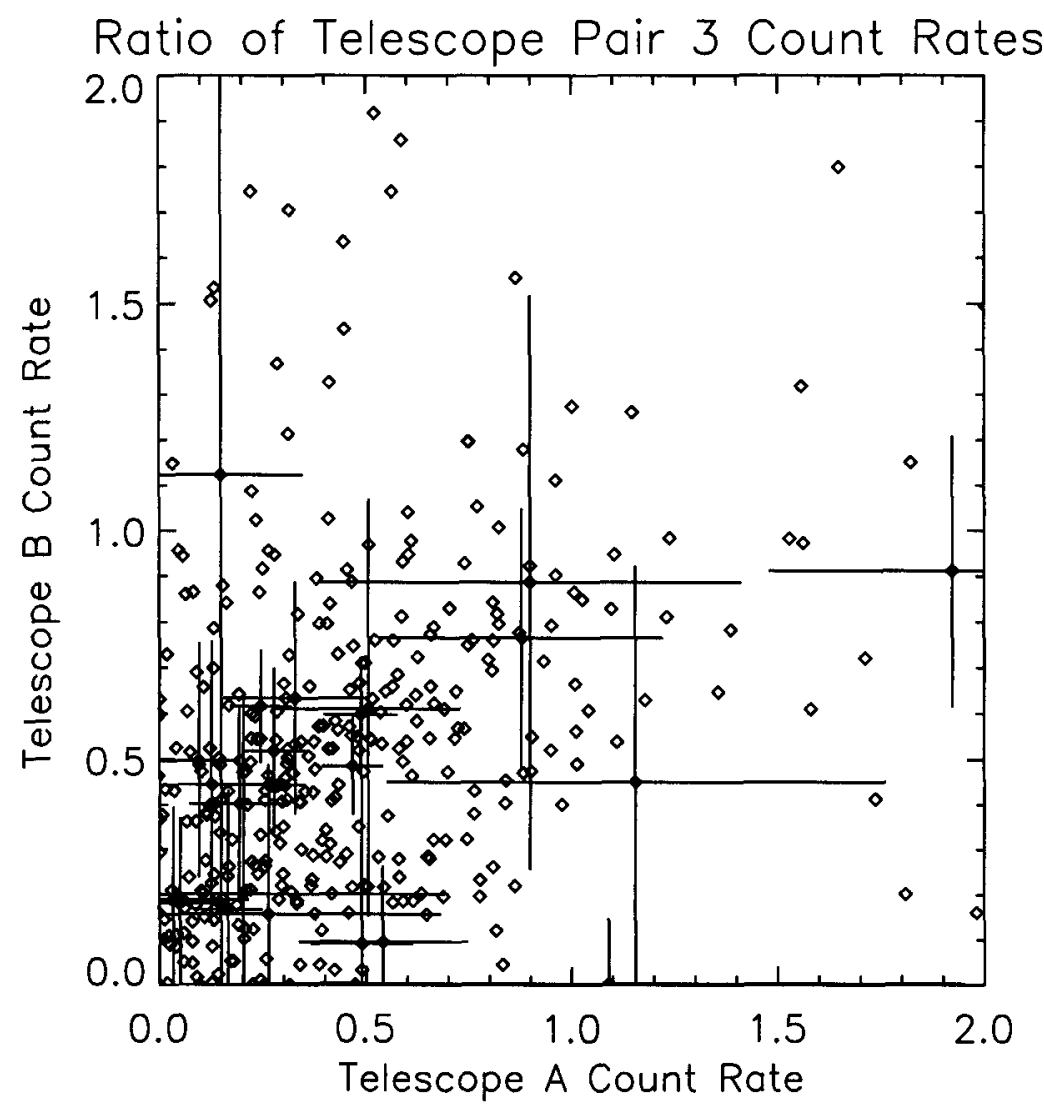

Figure 5. Rate ratio displayed as $3 A: 174 \AA$ vs. $3 B: 188 \AA$. Only points where both $3 A$ and $3 B$ had overlapping observations are plotted. Several representative error bars are shown. 


\section{Conclusions}

Alexis observations of the moon have high statistics and show interesting temporal and spectral variations. This data directly relates to processes occurring in the outer layers of the sun and may be a unique monitor of their temperature variations. Future work is required to improve the data quality and determine its value as a solar monitor.

The authors would like to thank the entire Alexis team for their hard work and dedication. This work is supported by the Department of Energy.

\section{REFERENCES}

Bearman, P. W. \& Graham, J. M. R. 1980, Vortex shedding from bluff bodies in oscillating flow: A report on Euromech 119, J. Fluid Mech., 99, 225

Bloch, J. J., ET AL. 1990, Design, Performance, and Calibration of the ALEXIS Ultrasoft Xray Telescopes, SPIE 1344, EUV, X-Ray, and Gamma-Ray Instrumentation for Astronomy

Edwards, B., Priedhorsky, W. C., \& SMITH, B. W. 1991, Expected Extreme Ultraviolet Spectrum of the Lunar Surface, Geophys. Res. Lett., 18, 2161

Gladstone, G. R., McDonald, J. S., Boyd, W. T., \& Bowyer, S. 1993, EUVE Photometric Observations of the Moon, Geoph. Res. Lett., 21(6), 461

Heroux L., Cohen, M., \& Higgins, J. E. 1974, Electron Densities Between 110 and 300 km Derived From Solar EUV Fluxes of August 23 1972, J. Geophys. Res., 29(34) 